\title{
A NOTE ON A LEMMA OF ZARISKI AND HIGHER DERIVATIONS
}

\author{
WEI-EIHN KUAN
}

\begin{abstract}
A sufficient condition is given for an a-adic complete ring $R$ to be a power series ring over a subring.
\end{abstract}

1. Introduction. We prove in this note the following theorem: Let $R$ be a ring and let $a$ be an ideal in $R$ such that $\bigcap_{i=0}^{\infty} a^{i}=0$ and $R$ is complete with respect to the $a$-adic topology. Assume that there exists a higher derivation $D=\left\{D_{i}\right\}_{i=0}^{\infty}$ of $R$ such that $D_{1}(x)=1$ for some $x \in a$. Let $E=D_{0}-x D_{1}+\cdots+(-1)^{n} x^{n} D_{n}+\cdots$. If $E(x)=0$, then there exists a subring $R_{1}$ of $R$ such that $R=R_{1}[[x]]$, and $x$ is analytically independent over $R_{1}$.

This result generalizes Zariski's original lemma [5, Lemma 4, p. 526], and [1, Theorem 6, p. 412], a version of Zariski's lemma when $R$ is of positive characteristic, and also removes the condition that $R$ is an integral domain as we mentioned at the end of [1, p. 414]. [5, Lemma 4, p. 256] played a very important role in the study of analytic product of an affine algebraic variety $V$ along a given subvariety $W$ of $V$ in A. Seidenberg's paper on differential ideals [4].

In the last section, we generalize a lemma of M. Miyanishi [2, p. 194] slightly, and give some remarks on his proofs of his lemma and Proposition 1.3 [2, p. 194].

2. Preliminaries. Throughout this note, all rings are commutative with identity. A derivation $D$ of a ring $R$ is an additive group homomorphism from $R$ to $R$ such that $D(a \cdot b)=a D b+b D a$ for all $a$ and $b$ in $R$. A higher derivation $D=\left\{D_{i}\right\}_{i=0}^{\infty}$ of a ring $R$ is a sequence of additive group homomorphisms from $R$ to $R$ such that

(1) $D_{0}=$ identity map on $R$, and

(2) $D_{n}(a \cdot b)=\sum_{i=0}^{n} D_{i}(a) \cdot D_{n-i}(b)$ for all $n=1,2, \cdots$, and for all $a$ and $b$ in $R$. (Note $D_{1}$ is always a derivation of $R$.) Leibniz formula.

Let $R$ be a ring and let $a$ be an ideal in $R$. Then $R$ has a topological structure with $\left\{\mathfrak{a}^{2}\right\}_{i=0}^{\infty}$ as the fundamental system of neighborhoods of the

Received by the editors February 26, 1973.

AMS (MOS) subject classifications (1970). Primary 13J10, 13J05, $13 \mathrm{~B} 10$. ring.

Key words and phrases. Higher derivations, derivations, a-adic ring, power series

(C) American Mathematical Society 1974 
zero in $R$. This is the so called a-adic topology in $R$. Both addition and multiplication are continuous in a-adic topology. $R$ is a Hausdorff space if and only if $\bigcap_{i=0}^{\infty} a^{i}=0$. A mapping $f: R \rightarrow R$ is continuous if there exists a subsequence $\left\{a^{v_{n}}\right\}_{n=0}^{\infty}$ of the sequence $\left\{a^{n}\right\}_{n=0}^{\infty}$ such that $f\left(a^{v_{n}}\right) \subset a^{n}$, for each natural number $n$.

Lemma 1. Let $R$ be a ring. Let $D=\left\{D_{i}\right\}_{i=0}^{\infty}$ be a higher derivation of $R$. Then

(1) For $a_{1}, \cdots, a_{n} \in R$, and for each natural number $m$,

$$
\begin{aligned}
D_{m}\left(a_{1} \cdots a_{n}\right)= & \sum_{i=1}^{n} a_{1} \cdots \hat{a}_{i} \cdots a_{n} D_{m}\left(a_{i}\right) \\
& +\sum_{i=1}^{m-1} D_{i}\left(a_{1} \cdots a_{n-1}\right) \cdot D_{m-i}\left(a_{n}\right) \\
& +\sum_{i=1}^{m-1} \sum_{j=2}^{n-1} a_{j+1} \cdots a_{n} D_{i}\left(a_{1} \cdots a_{j-1}\right) D_{m-i}\left(a_{j}\right),
\end{aligned}
$$

where $a_{1} \cdots \hat{a}_{i} \cdots a_{n}=a_{1} \cdots a_{i-1} a_{i+1} \cdots a_{n}$.

(2) For each ideal $a$ in $R$, and for each natural number $m, D_{m}\left(a^{n}\right) \subset a^{n-m}$ for each $\mathrm{a}^{n}$, i.e. $D_{m}$ is continuous with respect to the a-adic topology.

(3) For each ideal $a$ in $R$ and for each $x \in \mathfrak{a}$, let $E_{l}=\sum_{i=0}^{l}(-1)^{i} x^{i} D_{i}$ for each natural number $l$. $E_{l}\left(a^{n}\right) \subset a^{n}$ for each $a^{n}$, i.e. $E_{l}$ is a continuous additive group homomorphism of $R$ with respect to the a-adic topology.

Proof. Straightforward.

LEMMA 2. Let $R$ be a ring. Let $a$ be an ideal of $R$ such that $\bigcap_{i=0}^{\infty} a^{i}=0$. Assume $R$ is complete with respect to the a-adic topology. Let $x \in \mathfrak{a}$, and let $E_{l}=\sum_{i=0}^{l}(-1)^{i} x^{i} D_{i}$. Then the sequence $\left\{E_{l}\right\}_{l=0}^{\infty}$ is uniformly convergent, and $E=\sum_{l=0}^{\infty}(-1)^{i} x^{i} D_{i}=\lim _{l \rightarrow \infty} E_{l}$ is a continuous endomorphism of $R$.

Proof. The sequence $\left\{E_{l}(a)\right\}$ is a Cauchy sequence for each $a \in R$. In fact $E_{i}(a)-E_{j}(a) \in \mathrm{a}^{n}$ for $i, j>n$; i.e., for each given $\mathfrak{a}^{n}$, there exists a natural number $N(=n)$ such that $E_{i}(a)-E_{j}(a) \in a^{n}$ for $i, j>N$. Hence $\left\{E_{l}(a)\right\}$ converges in $R$. Since the natural number $N$ above is independent of $a \in R$, therefore $\left\{E_{l}\right\}_{l=0}^{\infty}$ converges uniformly in $R$. Hence $E=\lim _{l \rightarrow \infty} E_{l}$ is continuous. In fact for each $a^{n}$ and for each $a \in a^{n}$, there exists a natural number $N=n$ which is independent of $a$ such that $E(a)=\left(E(a)-E_{N}(a)\right)+$ $E_{.} \cdot(a) \in \mathfrak{a}^{n}$. Thus $E\left(\mathfrak{a}^{n}\right) \subset \mathfrak{a}^{n}{ }^{1}$

Since $E(a+b)=\lim _{l \rightarrow \infty} E_{l}(a+b)=\lim _{l \rightarrow \infty} E_{l}(a)+\lim _{l \rightarrow \infty} E_{l}(b)=E(a)+$ $E(b)$, therefore $E(a+b)=E(a)+E(b)$. Also for each natural number $l$,

I thank the referee for his remark that $E\left(a^{n}\right) \subset a^{n}$. 
$E_{l}(a) \cdot E_{l}(b) \equiv E_{l}(a b) \bmod \left(x^{l+1}\right)$. Thus $E_{l}(a) E_{l}(b)-E_{l}(a b) \in \mathfrak{a}^{l+1}$. Therefore

$$
\lim _{l \rightarrow \infty} E_{l}(a) \cdot E_{l}(b)=\lim _{l \rightarrow \infty} E_{l}(a b) \text {. }
$$

But $\lim _{l \rightarrow \infty} E_{l}(a) \cdot E_{l}(b)=\lim _{l \rightarrow \infty} E_{l}(a) \cdot \lim _{l \rightarrow \infty} E_{l}(b)$. So $E(a b)=E(a) E(b)$.

3. TheOREM 1. Let $R$ be a ring and let a be a proper ideal in $R$ such that $\bigcap_{i=0}^{\infty} a^{i}=0$, and $R$ is complete with respect to the a-adic topology. Assume that there exists a higher derivation $D=\left\{D_{i}\right\}_{i=0}^{\infty}$ of $R$ such that $D_{1}(x)=1$ for some $x \in a$. Let $E=D_{0}-x D_{1}+\cdots+(-1)^{n} x^{n} D_{n}+\cdots$. If $E(x)=0$, then there exists a subring $R_{1}$ of $R$ such that $R=R_{1}[[x]]$, and $x$ is analytically independent over $R_{1}$; i.e. if $\sum_{i=0}^{\infty} a_{i} x^{i}=0$ where $a_{i} \in R_{1}$ then $a_{i}=0$ for all $i=1,2, \cdots$.

Proof. $E(x)=0$ implies $E^{-1}(0)=R x$. Indeed, if $a \in E^{-1}(0)$, then $E(a)=0$; i.e. $\quad a-x D_{1}(a)+\cdots+(-1)^{n} x^{n} D_{n}(a)+\cdots=0$. Thus $a=$ $x D_{1}(a)+\cdots+(-1)^{n} x^{n} D_{n}(a)+\cdots$ is in $R x$. So $E^{-1}(0) \subset R x$. The other inclusion is obvious. Next, we observe that $E^{2}=E$. In fact for each $a \in R$,

and

$$
E(a)=a-x D_{1} a+\cdots
$$

$$
\begin{aligned}
E^{2}(a) & =E(a)-E\left(x D_{1} a+\cdots\right) \\
& =E(a)-E(x) \cdot E\left(D_{1} a-x D_{2} a+\cdots\right)=E(a),
\end{aligned}
$$

so $E^{2}(a)=E(a)$ for all $a \in R$. Let $R_{1}=E(R)$ then $E$ is an identity map on $R_{1}$. Let $a$ be an arbitrary element in $R$. $E(a)=a-x D_{1} a+\cdots+$ $(-1)^{n} x^{n} D_{n} a+\cdots$ implies that $a=E(a)+a_{1} x$ for some $a_{1} \in R$. Thus $a=E(a)+x E\left(a_{1}\right)+a_{2} x^{2}$ for some $a_{2} \in R$, and so on. Therefore we have $a=E(a)+x E\left(a_{1}\right)+\cdots+x^{n} E\left(a_{n}\right)+\cdots$ for some $a_{1}, a_{2}, \cdots, a_{n}, \cdots$ in $R$, and $a \in R_{1}[[x]]$. Hence $R=R_{1}[[x]]$. Finally we suppose $b_{0}+b_{1} x+\cdots+$ $b_{m} x^{n}+\cdots=0$ where $b_{0}, b_{1}, \cdots, b_{n}, \cdots$ are in $R_{1}$. We prove inductively $b_{0}=b_{1}=\cdots=b_{n}=\cdots=0$. Since $E$ is identity on $R_{1}, E\left(b_{0}+b_{1} x+\cdots+\right.$ $\left.b_{n} x^{n}+\cdots\right)=0$ implies $b_{0}=E\left(b_{0}\right)=0$. Assume $b_{0}=b_{1}=\cdots=b_{i}=0$. We have $b_{i+1} x^{i+1}+b_{i+2} x^{i+2}+\cdots=0$. By Lemma 1 and $D_{1}(x)=1$, we have $D_{n} x^{n} \equiv 1 \bmod (x)$ and $D_{n} x^{n+j} \equiv 0 \bmod (x)$ for all natural numbers $n$ and $j$. Thus

$$
\begin{aligned}
0 & =D_{i+1}\left(b_{i+1} x^{i+1}+b_{i+2} x^{i+2}+\cdots\right) \\
& =D_{i+1}\left(b_{i+1} x^{i+1}\right)+D_{i+1}\left(b_{i+2} x^{i+2}+\cdots\right) \\
& \equiv b_{i+1} D_{i+1}\left(x^{i+1}\right) \bmod (x) \equiv b_{i+1} \bmod (x) .
\end{aligned}
$$

Therefore $b_{i+1}+c_{i+1} x=0$ for some $c_{i+1} \in R$. So $0=E\left(b_{i+1}+c_{i+1} x\right)=b_{i+1}$. Therefore $x$ is analytically independent over $R_{1}$. 
If $R$ contains the field of rational numbers as a subring, then every derivation $D$ of $R$ gives rise to a higher derivation of $R$, namely, $\left\{D_{0}, D\right.$, $\left.D^{2} / 2 !, \cdots, D^{n} / n !, \cdots\right\}$ where $D^{n}$ is the $n$th successive derivation of $D$, and $D_{0}$ is the identity map in $R$. Let a be an ideal of $R$ such that $R$ is a complete Hausdorff space with respect to the a-adic topology. Assume $D x=1$ for some $x \in a$. Then the endomorphism $E=e^{-x D}=\sum(-1)^{n} x^{n} D^{n} / n$ ! always maps $x$ to zero. Let $R_{1}=E(R)$, then $D\left(R_{1}\right)=0$. Thus we have the following

COROLLARY 1. Let $R$ be a ring containing the field of rational numbers as a subring. Let a be an ideal in $R$ such that $R$ is a complete Hausdorff space with respect to the a-adic topology. Assume there exists a derivation $D$ of $R$ such that $D x=1$ for some $x \in a$. Then there exists a subring $R_{1}$ of $R$ such that (1) $D$ is zero on $R_{1}$ and (2) $R=R_{1}[[x]]$ and $x$ is analytically independent over $R_{1}$.

In the following, a semilocal (local) ring $\mathfrak{D}$ is a Noetherian ring with finitely many (unique) maximal ideals. Let $m$ be the intersection of the maximal ideals of $\mathfrak{D}$. It is well known that $\bigcap_{i=0}^{\infty} \mathfrak{m}^{i}=0$. In this case we use m-adic topology for $R$. As a corollary to Corollary 1 , we have the original lemma of Zariski.

COROLlaRY 2. Let $(\mathfrak{D}, \mathrm{m})$ be a complete semilocal ring of characteristic zero. Let $D$ be a derivation of $\mathfrak{D}$. Assume that there exists an element $x$ in $m$ of $\mathfrak{D}$ such that $D x$ is a unit in $\mathfrak{D}$. Then $\mathfrak{D}$ contains a ring $\mathfrak{D}_{1}$ of representatives of the (complete) semilocal ring $\mathfrak{D} / \mathfrak{D} x$ having the following properties: (a) $D$ is zero on $\mathfrak{D}_{1}$; (b) $x$ is analytically independent on $\mathfrak{D}_{1}$; (c) $\mathfrak{D}$ is the power series ring $\mathfrak{D}_{1}[[x]]$.

Proof. Replace $D$ by $(1 / D x) D$ and apply Corollary 1 .

We also get [1, Theorem 6, p. 412] as a corollary to the theorem,

Corollary 3. Let $(\mathfrak{D}, \mathrm{m})$ be a complete local ring. Let $x \in \mathrm{m}$ and let $D=\left\{D_{i}\right\}_{i=0}^{\infty}$ be a higher derivation of $\mathfrak{D}$ such that $D_{1} x$ is a unit in $\mathfrak{D}$, and $D_{i} x=0$ for $i>1$. Then there exists a subring $\mathfrak{D}_{1}$ of $\mathfrak{D}$ such that: (a) $\mathfrak{D}_{1}$ is a complete local ring, (b) $x$ is analytically independent over $\mathfrak{D}_{1}$, and (c) $\mathfrak{D}=\mathfrak{D}_{1}[[x]]$.

Proof. Let $D_{1} x=\varepsilon^{-1}$, where $\varepsilon$ is a unit in $\mathfrak{D}$. Replacing $\left\{D_{i}\right\}_{i=0}^{\infty}$ by $\left\{\varepsilon^{i} D_{i}\right\}_{i=0}^{\infty}$, we may assume $D_{1} x=1$. Since $D_{i} x=0$ for $i>1$, therefore $E x=x-x D_{1} x=0$ where $E=D_{0}-x D_{1}+\cdots+(-1)^{n} x^{n} D_{n}+\cdots$. Thus the theorem is applicable.

Remarks. (1) Theorem 1 and Corollary 1 hold under the assumption that $D_{1} x$ is a unit. The proofs are easily modified. 
(2) If $R$ has $a$ as its sole maximal ideal and is a complete Hausdorff space with respect to the a-adic topology, then $E(x)=0$ and $x \neq 0$ implies that $D_{1}(x)$ is a unit.

(3) If $R$ is a complete Hausdorff integral domain with respect to the a-adic topology, then $E(x)=0$ and $x \neq 0$ implies that $D_{1}(x)$ is a unit.

4. Though the following theorem could be easily proved by a similar technique used in the proof of Theorem 1, we would like to prove it as a corollary to Corollary 1.

THEOREM 2. Let $R$ be a ring containing the field of rational numbers as a subring. Let $a$ be an ideal in $R$ such that $\bigcap_{i=0}^{\infty} \mathfrak{a}^{i}=0$. Assume that there exists a derivation $D$ of $R$ such that (1) for each $\gamma \in R, D^{i}(\gamma)=0$ for sufficiently large $i$, and (2) $D(x)=1$ for some $x \in \mathfrak{a}$. Then (a) there exists a subring $R_{1}$ of $R$ such that $R=R_{1}[x]$ and $x$ is algebraically independent over $R_{1}$; (b) $D$ is trivial on $R_{1}$.

Proof. Let $\hat{R}$ be the completion of $R$ with respect to the a-adic topology. Then $\hat{R}$ is a complete Hausdorff space with respect to the topology defined by the filtration $\hat{a} \supset\left(a^{2}\right)^{\wedge} \supset \cdots \supset\left(a^{n}\right)^{\wedge} \supset \cdots$, where $\left(a^{i}\right)^{\wedge}$ is the closure of $a^{i}$ in $\hat{R}$. Thus $\bigcap_{i=0}^{\infty}\left(a^{i}\right)^{\wedge}=0$. Note that $\left(a^{i}\right)^{\wedge} \supsetneqq a^{i} \hat{R}$ in general and equality holds if $a$ is finitely generated. Let $\gamma_{1}, \gamma_{2}, \cdots, \gamma_{n}, \cdots$ be a Cauchy sequence in $R$; Lemma 1 implies that $\left\{D^{j}\left(\gamma_{i}\right)\right\}_{i=0}^{\infty}$ is also a Cauchy sequence for each $j$. Define $\hat{D}^{j}(\gamma)=\lim _{i \rightarrow \infty} D^{j}\left(\gamma_{i}\right)$. Then it is easy to check that $\left\{\hat{D}_{0}, \hat{D}, \cdots, \hat{D}^{n} / n !, \cdots\right\}$ forms a higher derivation in $\hat{R}$. Moreover $\hat{D}^{j}\left(\mathfrak{a}^{i}\right)^{\wedge} \subset\left(\mathfrak{a}^{i-j}\right)^{\wedge}$. Indeed let $\hat{\gamma} \in\left(\mathfrak{a}^{i}\right)^{\wedge}$ and let $\gamma_{1}, \cdots$, $\gamma_{n}, \cdots$ be a sequence in $\mathfrak{a}^{i}$ such that $\lim _{n \rightarrow \infty} \gamma_{n}=\hat{\gamma}$. Then $\hat{D}^{j}(\hat{\gamma})=$ $\lim _{n \rightarrow \infty} D^{j}\left(\gamma_{n}\right)$. Since $D^{j}\left(\gamma_{n}\right) \in \mathfrak{a}^{i-j}$, therefore $\hat{D}^{j}(\hat{\gamma}) \in\left(\mathfrak{a}^{i-j}\right)^{\wedge}$. Lemma 1 and Lemma 2 are easily verified. Since the kernel of the natural ring homomorphism from $R$ to $\hat{R}$ is $\bigcap_{i=0}^{\infty} a^{i}=0, R$ is viewed as a subring of $\hat{R}$. $\hat{D}^{i}$ restricted to $R$ is $D^{i}$ so $\hat{D} x=1$ and

$$
\begin{aligned}
E(x) & =x-(x / 1 !) \hat{D}(x)+\cdots+(-1)^{n}\left(x^{n} / n !\right) \hat{D}^{n}(x)+\cdots \\
& =x-(x / 1 !) D(x)+\left(x^{2} / 2 !\right) D^{2}(x)+\cdots+(-1)^{n}\left(x^{n} / n !\right) D^{n}(x)+\cdots \\
& =0 .
\end{aligned}
$$

Thus it follows from Theorem 1 that $\hat{R}=\hat{R}_{1}[[x]]$, where $\hat{R}_{1}$ is a subring of $\hat{R}$ and $x$ is analytically independent over $\hat{R}_{1}$. Let $R_{1}=R \cap \hat{R}_{1}$. Then $R=R_{1}[x]$. Indeed, let $\gamma \in R$. Then $\gamma=a_{0}+a_{1} x+\cdots+a_{n} x^{n}+\cdots$ where $a_{i} \in \hat{R}_{1}$ for all $a_{i}$. Since there exists a natural number $N$ such that $D * v(\gamma)=0$. Therefore

$$
0=\hat{D}^{N}(\gamma)=N ! a_{N}+\frac{1}{2}(N+1) ! a_{N+1} x+\cdots .
$$

Hence $a_{i}=0$ for all $i \geqq N$, and $\gamma=a_{0}+a_{1} x+\cdots+a_{N-1} x^{N-1}$. It follows that $R \subset \hat{R}_{1}[x]$. Applying $D^{v-1}$ to $\gamma$, we have $D^{N-1}(\gamma)=(N-1) ! a_{N-1}$. 
Therefore $a_{N-1} \in R$. Applying $D^{N-2}$ to $\left(\gamma-a_{N-1} x^{N-1}\right)=a_{0}+a_{1} x+\cdots+$ $a_{N-2} x^{N-2}$, we get $a_{N-2} \in R$ and so on. Consequently, $a_{0}, \cdots, a_{N-1}$ are all in $\hat{R}_{1} \cap R=R_{1}$. So $R=R_{1}[x]$, and $x$ is of course algebraically independent over $R_{1} \cdot$ (b) follows from Corollary 1 .

We would like to thank Professor M. Miyanishi for communicating to us the following result which we also observed independently.

Proposition 1. Let $R$ be an integral domain of characteristic 0 . Assume there is a derivation of $R$ such that $D^{i}(a)=0$ for each $a \in R$ and for sufficiently large $i$. Then $D x=0$ for all units $x$ in $R$.

Proof. Let $x$ be a unit in $R$, and let $y \in R$ be such that $x y=1$. Then $x D y+y D x=0$. Suppose $D x \neq 0$. Thus $D y \neq 0$. Let $i$ be the natural number such that $D^{i} x=0$ and $D^{m} x \neq 0$ for $m<i$, also let $j$ be the natural number such that $D^{j} y=0$ and $D^{p} y \neq 0$ for $p<j$. By Leibniz's formula,

$$
0=D^{n}(x y)=\sum_{k=0}^{n}\left(\begin{array}{l}
n \\
k
\end{array}\right) D^{k}(x) D^{n-k}(y) .
$$

Taking $n=i+j-2$ and assuming $i \leqq j$ we get $D^{j-1}(x) \cdot D^{i-1}(y)=0$. Hence either $D^{i-1}(x)=0$ or $D^{j-1}(y)=0$, a contradiction.

Proposition 1 completes the proof of [2, Lemma 1.4, p. 194]. One could not use [2, Proposition 1.4, p. 194] to yield a proof to the last part of [2, Proposition 1.3, p. 193]. But Proposition 1 corrects that part of the proof.

[4, Theorem 5, p. 30] locates differential ideals; [2, Proposition 1.3, p. 193] becomes interesting, we give it a slight generalization.

THEOREM 3. Let $R$ be an integral domain of characteristic 0 with a unique maximal ideal $\mathrm{m}$ such that $\bigcap_{i=0}^{\infty} \mathrm{m}^{i}=0$, i.e. $(R, \mathrm{~m})$ is a local domain which may not be Noetherian. If there is a derivation $D$ of $R$ such that $D^{i}(a)=0$ for each $a \in R$ and for sufficiently large $i$, then $\mathrm{m}$ is differential, i.e. $D(\mathrm{~m}) \subset \mathrm{m}$, and $\bar{D}$ induced by $D$ on $R / \mathrm{m}$ is trivial.

Proof. Suppose $D m \notin m$. Then there is $x \in m$ such that $D x=u^{-1}$, where $u$ is a unit in $R$. Then $u D x=1$. Replacing $D$ by $u D$, we have $(u D)^{i}(a)=u^{i} D^{i}(a)$ by Proposition 1 . Thus $(u D)^{i}(a)=0$ for sufficiently large $i$. It follows from Theorem 2 that $R=R_{1}[x]$, a contradiction. The last part follows from Proposition 1.

Observing the fact that in a polynomial ring $A[x]$ the units in $A[X]$ are of the form $a_{0}+a_{1} x+\cdots+a_{n} x^{n}$ such that $a_{0}$ is a unit in $A$ and $a_{2}, \cdots, a_{n}$ are nilpotent in $A$, we give two examples countering Proposition 1 when $R$ is not an integral domain. 
EXAMPLE 1. Let $R=Z /(4)[X]$, where $Z$ is the domain of integers and $X$ is an indeterminate over $Z /(4)$. Let $D$ be a derivation of $R$ such that $D X=1+2 X+2 X^{2} .(D X)^{2}=1, D^{2} X=2 \neq 0, D^{i}(a)=0$ for each $a \in R$ and for large integers $i$.

EXAMPLE 2. Let $R=(Q[t])[X]$, where $Q$ is the field of rational numbers, $t^{2}=0$ and $X$ is an indeterminate over $Q[t]$. Let $D$ be a derivation of $R$ such that $D X=1+t X$. Then $D X$ is a unit $((D X) \cdot(1-t X)=1)$ $D^{2} X=t \neq 0$, and $D t=0, D^{i}(a)=0$ for each $a \in R$ and for large $i$.

In the setting of Theorem 2 , when $R$ is an integral domain, if there is a derivation $D$ such that $D X$ is a unit $u$ for some $x \in a$, then there is a $y \in \mathfrak{a}(y=x / u)$ such that $D y=1$. What can one say in a more general case? Both Example 1 and Example 2 give negative answers. Using an idea of Professor M. Rosenlicht [3, Theorem 1, p. 721] we prove the following theorem.

THEOREM 4. Let $R$ be a ring, which contains the field of rational numbers, with an ideal a such that $\bigcap_{i=0}^{\infty} a^{i}=0$ and $R$ is complete with respect to the a-adic topology. Assume there is a derivation $D$ of $R$ such that $D x$ is a unit for some $x \in \mathfrak{a}$. Then there exists an element $y \in \mathfrak{a}$ such that $D y=1$.

Proof. If $D x$ is a unit then $D(x / D x)-1 \in R x \subset \mathrm{a}$. If we can construct a Cauchy sequence $\left\{x_{0}=x / D x, x_{1}, \cdots, x_{n}, \cdots\right\}$ such that $x_{i} \in x R$ and $D x_{i}-1 \in R x^{i+1}$, then putting $y=\lim _{i \rightarrow \infty} x_{i}$, and since $a$ is also closed, we have $y \in \mathfrak{a}$ and $D y=\lim _{i \rightarrow \infty} D x_{i}=1$. The proposed construction goes inductively as follows: Since $D\left(R x^{i+1}\right) \subset R x^{i}, D$ induces a surjective $R$-homomorphism $\bar{D}^{(i)}: R x^{i+1} \rightarrow R x^{i} / R x^{i+1}$ such that

$$
\bar{D}^{(i)}\left(x^{i+1}\right)=(i+1) x^{i}+R x^{i+1} .
$$

Therefore there exists $z_{i} \in R x^{i+1}$ such that

$$
D z_{i} \equiv D x_{i-1}-1 \bmod \left(R x^{i+1}\right)
$$

for $i=1,2, \cdots$. Thus $D\left(x_{i-1}-z_{i}\right)-1 \in R x^{i+1}$. Putting $x_{i}=x_{i-1}-z_{i}$, we have a sequence $\left\{x_{0}=x / D x, x_{1}, x_{2}, \cdots\right\}$ such that $D x_{i}-1 \in R x^{i+1} \subset$ $\mathfrak{a}^{i+1}$ for $i=0,1,2, \cdots$. For a given $\mathfrak{a}^{n}$, there exists a positive integer $N$ $(=n)$ such that for $i, j>N, x_{i}-x_{j} \in \mathfrak{a}^{n}$. Therefore $\left\{x_{0}, x_{1}, \cdots\right\}$ is a Cauchy sequence as desired.

ADDED IN PROOF. The author recently discovered that Theorem 2 can be derived from Taylor's lemma, see Y. Nouaze and P. Gabriel's Idéaux premiers de l'algèbre enveloppante d'une algèbre de Lie nilpotente, J. Algebra 6 (1967), 77-99. 


\section{REFERENCES}

1. W. C. Brown and W. Kuan, Ideals and higher derivations in commutative rings, Canad. J. Math. 24 (1972), 400-415. MR 45 \#3388.

2. M. Miyanishi, Some remarks on the actions of additive group schemes. J. Math. Kyoto Univ. 10 (1970), 189-205. MR 43 \#7437.

3. M. Rosenlicht, Canonical forms for local derivations, Pacific J. Math. 42 (1972), 721-732.

4. A. Seidenberg, Differential ideals in rings of finitely generated type, Amer. J. Math. 89 (1967), 22-42. MR 35 \#2902.

5. O. Zariski, Studies in equisingularity. I. Equivalent singularities of plane algebroid curves, Amer. J. Math. 87 (1965), 507-536. MR 31 \#2243.

Department of Mathematics, Michigan State University, East Lansing, MichigAN 48823 